\title{
Thrombotic superior vena caval obstruction after repair of tetralogy of Fallot
}

\author{
A. J. MEARNS, J. A. DAVIES, C. A. HOGgARD, AND D. A. WATSON \\ From Killingbeck Hospital, Leeds, UK
}

Mearns, A. J., Davies, J. A., Hoggard, C. A., and Watson, D. A. (1977). Thorax, 32, 623-627.

Thrombotic superior vena caval obstruction after repair of tetralogy of Fallot. A case of tetralogy of Fallot is described, in which thrombotic obstruction of the superior vena cava

developed after total correction. Partial thrombolysis was achieved with streptokinase therapy.

Obstruction of the superior vena cava is usually related to underlying malignant disease. Thrombotic obstruction of the superior vena cava is uncommon (Lokich and Goodman, 1975) and has not apparently been described previously as a complication of repair of tetralogy of Fallot. While the effectiveness of streptokinase in thrombus dissolution has been reported from studies on venous and arterial occlusion (Leveen and Diaz, 1972) and on pulmonary embolism (Tibbutt et al., 1974), streptokinase has rarely been used in the treatment of thrombotic superior vena caval obstruction (Effeney et al., 1973; Williams and Demos, 1974).

\section{Case report}

Acyanotic tetralogy of Fallot was first diagnosed in the patient at $6 \frac{1}{2}$ years of age. At 19 years her effort tolerance was grade 2 in the New York Heart Association classification. The only other evidence of impaired cardiac function was the development of slight cyanosis on exertion. She was admitted for total surgical correction because of the prospect of marriage and pregnancy.

Cardiac catheterisation confirmed the diagnosis. Pulmonary valve stenosis with infundibular obstruction was demonstrated, with right ventricular systolic pressure of $25 \mathrm{mmHg}$. There was a right-to-left shunt of $2.5: 1$ through an infracristal ventricular septal defect and oxygen saturation in the aorta was reduced to $86 \%$.

At operation a large infracristal septal defect was repaired with a Dacron patch through a right ventriculotomy. Hypertrophied muscle bands were excised from the outflow tract of the right ventricle. Exploration of the pulmonary valve showed a diaphragm with an eccentric crescent-shaped orifice. Obstruction was relieved by incision of the commissures of the valve to allow passage of a $9 \mathrm{~mm}$ sound.

Measurement of cardiac function at the end of the period of bypass was satisfactory. The right ventricular systolic pressure was $40 \mathrm{mmHg}$, pulmonary artery systolic pressure $20 \mathrm{mmHg}$, and left ventricular systolic pressure $110 \mathrm{mmHg}$. Eight hours later complete heart block developed which reverted to sinus rhythm after ventricular pacing for eight hours. Cardiac function remained good and the central venous pressure catheter was removed three days later. The central venous pressure was maintained at a maximum of $16 \mathrm{mmHg}$ but showed a V-wave consistent with mild tricuspid incompetence.

Recovery was uneventful until the third day when she developed clinical signs of a right pleural effusion. This increased and a small effusion developed in the left pleural space. Digoxin and diuretics did not limit these effusions and so both were aspirated, producing a total of $930 \mathrm{ml}$ of serous fluid.

On the 13th day, signs of subacute cardiac tamponade developed with a paradoxical pulse and poor peripheral circulation. The white blood cell count rose to $27.0 \times 10^{9} / 1$. Pericardial aspiration yielded $500 \mathrm{ml}$ of serosanguinous fluid. Her condition then improved sufficiently to allow weekend leave on the 22 nd day.

Twenty-four hours later she was readmitted because of the sudden development of fullness of the neck and oedema of the face, arms, and left breast. Jugular venous pressure was raised to the angle of the jaw. Obstruction of the superior vena cava was diagnosed but, because of the pos- 
sibility of venous obstruction from pericardial effusion, the pericardium was again aspirated. Though $600 \mathrm{ml}$ of fluid was withdrawn, it was not under pressure and removal did not affect the clinical signs.

Cardiac catheterisation was again performed via the right median basilic vein, and this showed the repair to be satisfactory. There was no evidence of shunting and the pressures were: pulmonary wedge $17 \mathrm{mmHg}$, right ventricular systolic $45 \mathrm{mmHg}$, and mean right atrial $16 \mathrm{mmHg}$. Simultaneous bilateral upper limb venography (right-sided injection through catheter in median basilic vein and left-sided injection through venepuncture in cubital fossa) showed extensive thrombosis of the innominate and subclavian veins, and of the superior vena cava (Fig. 1). Venography of the inferior vena cava was normal.

Surgical thrombectomy was considered to be unsuitable for such extensive thrombus and accordingly therapy with streptokinase was started. A loading dose of 600000 units was given, followed by 100000 units hourly for five days. Therapy was controlled by estimation of the thrombin time every 12 hours. At the end of the treatment with streptokinase therapy was continued with warfarin. During the period of infusion, the clinical signs of superior vena caval obstruction seemed to improve. Upper limb venography at the end of the infusion showed a decrease in collaterals and partial recanalisation of the innominate veins (Fig. 2). Her cardiac failure was aggravated, and gross dependent oedema and hepatosplenomegaly with ascites developed, which responded slowly to treatment with digoxin and diuretics. In view of $\overrightarrow{\vec{O}}$ the heart failure, pyrexia of $39^{\circ} \mathrm{C}$ (ascribed partly to streptokinase), and development of splinter hae- $\frac{\bar{\sigma}}{\sigma}$ morrhages in the nail beds, antibiotic therapy was $\frac{\bar{\sigma}}{2}$ instituted because of the possibility of endocarditis. $\mathbb{\nabla}$ Immunoglobulin assay supported the diagnosis of infection. There were raised levels of IgM at os $580 \mathrm{IU} / \mathrm{ml}$ and reduced $\mathrm{C}_{3}$ complement of $78 \mathrm{mg} / \vec{\circ}$ $100 \mathrm{ml}$. Because exhaustive bacteriological investi- $\overrightarrow{\vec{\omega}}$ gations had not isolated any organism, intravenous gentamicin and penicillin were administered $\overrightarrow{\vec{*}}$ empirically. Metronidazole was also given to cover $\underset{\omega}{\dot{\omega}}$ infection with heparinase-producing Bacteroides $\mathrm{N}$ which may have been related to the thrombotic or episode.

She made a slow recovery thereafter and was finally discharged from hospital nine weeks after the operation. Three months later, digoxin and diuretic therapy were stopped without recurrence of heart failure, though signs of incompetence of $\frac{}{0}$ pulmonary and tricuspid valves were present. There had also been some development of col- $\vec{\varphi}$ lateral veins over both clavicles, and venograms $\mathcal{Y}$ showed appearances essentially unaltered from those in Figure 2.

At present she is symptom free and has returned to work as a secretary.

\section{Discussion}

Venous thrombotic disease frequently complicates Fallot's tetralogy, but nearly always in cyanotic patients with secondary polycythaemia. This patient was cyanosed on exertion only, and

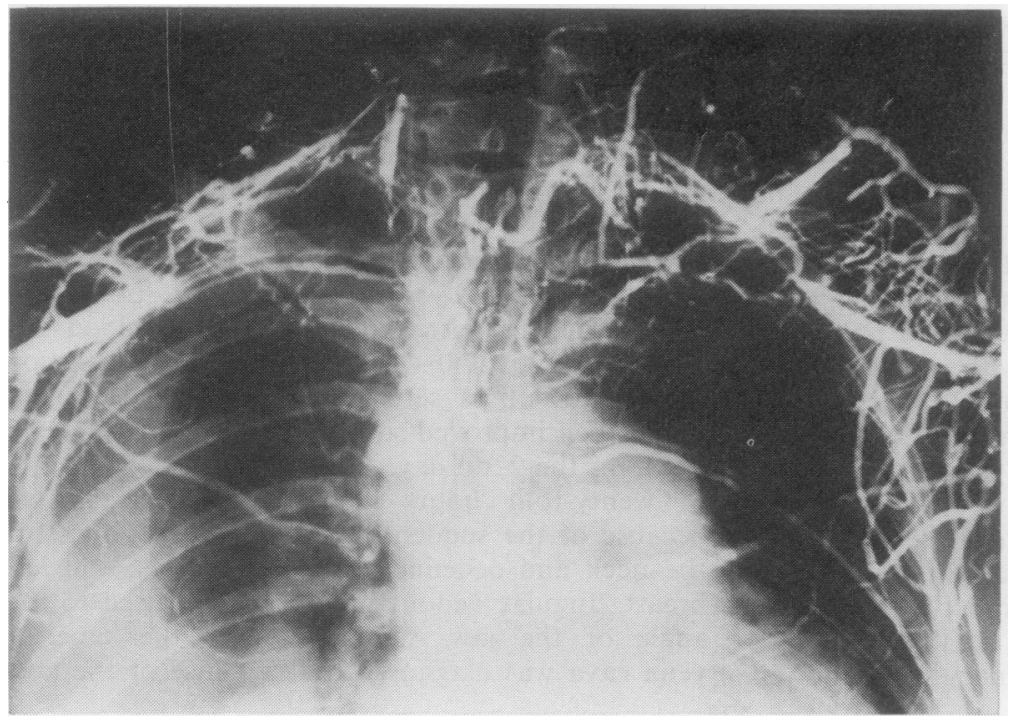

Fig. 1 Before thrombolytic therapy, showing absence of both subclavian and innominate veins. Filling of the superior vena cava is not seen, but there is extensive collateral flow, involving the paravertebral venous plexus, the intercostal veins, and both external jugular veins. 


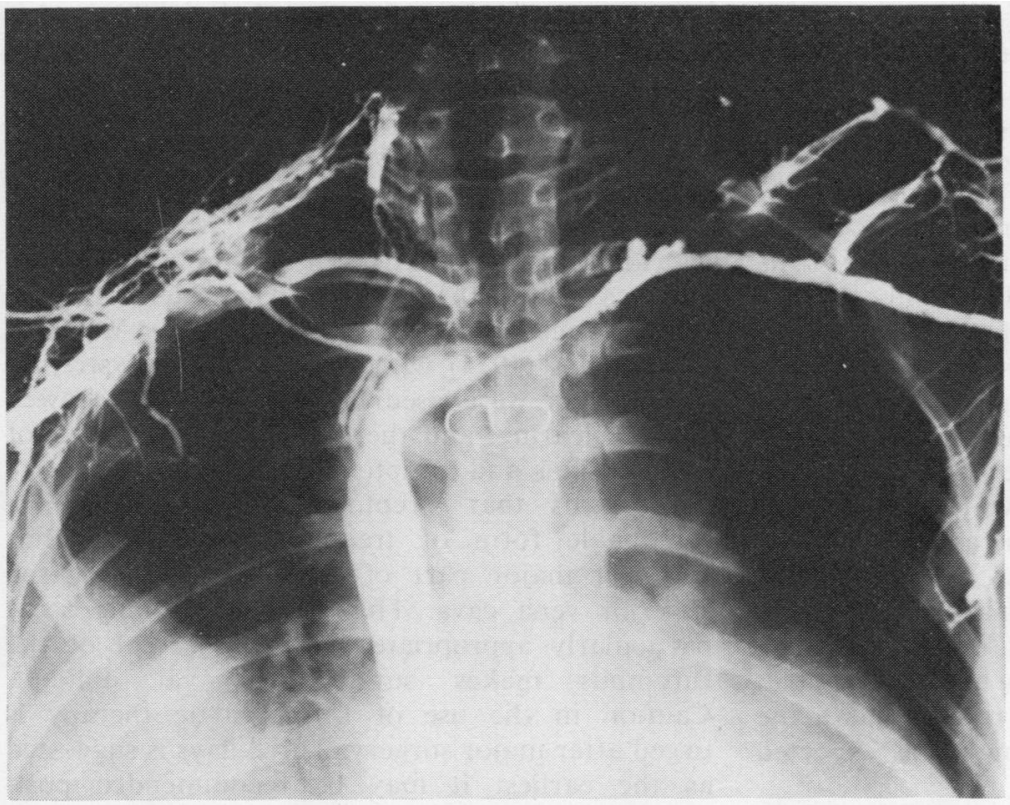

Fig. 2 After four days'

thrombolysis. Shows recanalisation of the left subclavian and innominate vein and good flow through the superior vena cava. There is evidence of mural thrombus at the junction of the right innominate vein and the superior vena cava. The collateral flow is much reduced although the right external jugular vein still opacifies.

the haematocrit never exceeded $45 \%$. Though thrombotic obstruction of the superior vena cava has been described in polycythaemia vera (Walsh et al., 1957), clearly factors other than increase in red cell mass are operative in these patients.

Total surgical correction of tetralogy of Fallot requires cannulation of the superior vena cava through the atrial appendage, the cannula being held in place by an encircling tape and snare. Routine cannulation of the superior vena cava via the anterior aspect of the right atrial appendage with a $7 \mathrm{~mm}$ polythene venous catheter was effected without difficulty. There were no problems with the nylon tapes $(5 \mathrm{~mm}$ flat) or the rubber snare. The cannula and snare were removed after bypass without incident.

The original subacute tamponade never reappeared although the pericardium was aspirated on readmission to exclude the possibility of intrapericardial compression of the superior vena cava from recurrence of the pericardial effusion. There were no signs of superior vena caval obstruction during the subacute tamponade treated 10 days earlier.

While trauma to the venous intima might be expected, thrombotic occlusion of the superior vena cava as a result is not a recognised complication. Thrombosis of the superior vena cava is known to result from the use of central venous pressure catheters, particularly when they are used for periods longer than seven days (Henzel and Deweese, 1971). In this patient a No. $16 \mathrm{EZ}$ catheter was inserted percutaneously from the left antecubital fossa on the morning of the operation. It remained in place until the third postoperative day, the signs of superior vena caval occlusion appearing only on the 23rd day. None of the drugs infused through it (potassium chloride, penicillin, diazepam, and papaveretum) is particularly likely to provoke thrombosis.

One factor in the development of the thrombosis may have been the persistently raised central venous pressure resulting from the period of subacute tamponade. Late tamponade is an infrequent complication of open-heart surgery and usually, as in this case, requires treatment by aspiration. However, although pericardial effusion may be associated with superior vena caval obstruction, this has been reported only with the malignant pericardial effusion of advanced neoplastic disease (Matin et al., 1970).

The problem of pleural effusion is not well covered in the literature. Our local experience suggests that right pleural aspiration is needed in $40 \%$ of corrections of Fallot's tetralogy, and bilateral aspiration in $10 \%$. We have not needed to aspirate the left chest alone for postoperative pleural effusion. Cleland et al. (1969) mention the problem of bilateral effusions and suggest that the causes are not known but may be associated with raised central venous pressure, increased pulmonary blood flow, or embolic venous obstruction.

Congestive cardiac failure after repair of 
Fallot's tetralogy is common; Clayman et al. (1975) described a $50 \%$ incidence in patients without an outflow tract patch.

The mild tricuspid incompetence was probably due to disturbance of the function of the papillary muscle of the conus. This was partially mobilised by cristal excision and had to be included in the margin of the patch because of its proximity. Inferior vena caval thrombosis was excluded at catheterisation and was unlikely to have progressed because the streptokinase was started immediately and shown to have had effect within four hours. In our patient the central venous pressure was never above $16 \mathrm{mmHg}$ at any stage. The pleural effusion shown in Fig. 1 may be the recurrent phenomenon associated with Fallot's tetralogy (Cleland et al., 1969) or may have been due to occult pulmonary emboli from the extensive superior vena caval obstruction. The superior vena cava is shown to be obstructed in both Fig. 1 and Fig. 2 in the region where the lymph from the right chest might be expected to drain.

Postoperative infection may have contributed in this case to the development of the thrombosis. Bacteroides fragilis, one of the organisms considered as a cause of infection in this patient, produces heparinase and is frequently associated with thromboembolic complications (Bodner et al., 1970). Strong presumptive evidence of infection existed here, although cultures of blood and aspirates for bacteria and fungi were persistently negative, as were the routine cultures of all catheter tips removed from the patient (Freeman, 1976). The mechanism by which infections predispose to thrombosis is ill-understood but interaction is known to occur between blood platelets and viruses, bacteria and antigen-antibody complexes (Mustard and Packham, 1975). This may result in platelet aggregate formation and release of procoagulant material from platelets. Antigen-antibody complexes may themselves initiate the contact phase of blood coagulation.

The development of thrombosis in this patient was probably multifactorial and related to the surgical procedure, trauma from the intravenous catheters, slow venous return, and infection.

Therapy with streptokinase appears to have been beneficial with evidence of clinical and radiological improvement, though extensive thrombus remained at the end of the treatment period. It is likely that the apparent changes between the first and second venograms were the result of the streptokinase infusion as no further improvement was seen on the third venogram carried out after six months of warfarin therapy.
The pattern of partial thrombolysis obtained is $\overrightarrow{\vec{F}}$ similar to the result obtained by Effeney and his colleagues (1973), who treated thrombosis of a superior vena caval graft with streptokinase. $\bar{c}$ Fibrinolytic therapy has also given encouraging results as an adjunct to therapy of malignant obstruction of the superior vena cava (Salsali and is Cliffton, 1968). A patient in whom the superior $\overrightarrow{0}$ vena cava was thrombosed from retention of a $\vec{\overrightarrow{ }}$ broken pacemaker wire was treated by Williams $\vec{\omega}_{\vec{T}}$ and Demos (1974) with smaller doses of strepto- $\vec{\partial}$ kinase than were used here. Venograms showed $x$ partial clearing, and there was complete relief of clinical signs and symptoms.

It seems that streptokinase may provide a reasonable form of treatment when thrombus forms a major part of the obstruction of the superior vena cava. This treatment would seem $\vec{a}$ particularly appropriate when the extent of the thrombus makes surgical removal difficult. $\frac{0}{0}$ Caution in the use of thrombolytic therapy is $\stackrel{\mathbb{N}}{-}$ urged after major surgery. Three days is suggested $\vec{\theta}$ as the earliest it may be recommended post- $\forall$ operatively (Browse, 1974), although it has been used as early as 24 hours (Fletcher et al., 1959).

A possible explanation of the sequence of events in this patient is one of initial serous pleural effusion after correction of Fallot's tetralogy and $\stackrel{\circ}{\mathbb{D}}$ then an insidious endovascular thrombosis of the $\stackrel{\square}{a}$ superior vena cava and its branches. The earliest obstruction was to the lymph drainage of the pericardium, followed by that from the right pleura, with possibly some embolisation of the right lung. Ultimately there was complete thrombosis of the superior vena cava and both innominate veins. This hypothesis is supported by evidence of incomplete clearing of the thrombus as streptokinase is thought to be ineffective in clearing thrombi more than 3 days old (Browse, 1974).

\section{References}

Bodner, S. J., Koenig, M. G., and Goodman, J. S. $\mathbb{N}$ (1970). Bacteremic Bacteroides infections. Annals $\Omega$ of Internal Medicine, 73, 537-544.

Browse, N. (1974). Advances in management of deep NN vein thrombosis. Modern Medicine of Great Britain, $\omega$ 19, 375-382.

Clayman, J. A., Ankeney, J. L., and Liebman, J. (1975). Results of complete repair of Tetralogy of $\Phi$ Fallot in 156 consecutive patients. American Journal $\stackrel{?}{-}$ of Surgery, 130, 5, 601-607.

Cleland, W. P., Goodwin, J., McDonald, L., and Ross, D. (1969). Medical and Surgical Cardiology, p. 766. Blackwell Scientific Publications, Oxford.

Effeney, D. J., Windsor, H. M., and Shanahan, M. X. (1973). Superior vena cava obstruction: resection - 
and bypass for malignant lesions. Australian and New Zealand Journal of Surgery, 42, 231-237.

Fletcher, A. P., Alkjaersig, N., and Sherry, S. (1959). The maintenance of a sustained thrombolytic state in man. 1. Induction and effects. Journal of Clinical Investigation, 38, 1096.

Freeman, R. (1976). Microbiological aspects of openheart surgery: diagnosis and management. In Current Techniques in Extra-corporeal Circulation, edited by M. I. Ionescu and G. H. Wooler, pp. 369395. Butterworths, London.

Henzel, J. H., and Deweese, M. S. (1971). Morbid and mortal complications associated with prolonged central venous cannulation. American Journal of Surgery, 121, 600-605.

Leveen, H. H., and Diaz, C. A. (1972). Venous and arterial occlusive disease treated by enzymatic clot lysis. Archives of Surgery, 105, 927-936.

Lokich, J. J., and Goodman, R. (1975). Superior vena cava syndrome. Journal of the American Medical Association, 231, 58-61.

Matin, P., Ray, G., and Kriss, J. P. (1970). Combined superior vena cava obstruction and pericardial effusion demonstrated by radioisotopic angiocardiography. Journal of Nuclear Medicine, 11, 78-80.
Mustard, J. F., and Packham, M. A. (1975). Platelets, thrombosis and drugs. Drugs, 9, 19-76.

Salsali, M., and Cliffton, E. (1968). Superior vena caval obstruction with lung cancer. Annals of Thoracic Surgery, 6, 437-442.

Tibbutt, D. A., Davies, J. A., Anderson, J. A., Fletcher, E. W. L., Hamill, J., Holt, J. M., Lea Thomas, M., Lee, G. de J., Miller, G. A. H., Sharp, A. A., and Sutton, G. C. (1974). Comparison by controlled clinical trial of streptokinase and heparin in treatment of life-threatening pulmonary embolism. British Medical Journal, 1, 343-347.

Walsh, G. C., Norton, G. I., Baird, M. M., and Robertson, R. (1957). Idiopathic thrombosis of superior vena cava. Canadian Medical Association Journal, 76, 292-295.

Williams, D. R., and Demos, N. J. (1974). Thrombosis of superior vena cava caused by pacemaker wire and managed with streptokinase. Journal of Thoracic and Cardiovascular Surgery, 68, 134-137.

Requests for reprints to: A. J. Mearns, FRCS, Killingbeck Hospital, Leeds, UK. 